\title{
Goals of care: a clinical framework for limitation of medical treatment
}

\begin{abstract}
Robyn L Thomas MBBS, FRACGP, FRAChPM Specialist in Palliative Medicine ${ }^{1}$

Mohamed Y Zubair Physician in General and Palliative Medicine ${ }^{2}$

Barbara Hayes MBBS, FAChPM, PhD Palliative Care Physician ${ }^{3}$

Michael A Ashby MD, FRACP, FFPMANZCA Director of Palliative Care ${ }^{1}$

1 Royal Hobart Hospital, Hobart, TAS.

2 Royal Darwin Hospital, Darwin, NT.

3 Advance Care Planning Program, Northern Health, Melbourne, VIC.

michael.ashby@

dhhs.tas.gov.au
\end{abstract}

doi: 10.5694/mjal4.00623

Medical decision making is based on determining the GOC for the patient. The patient's situation is assigned to one of three phases of care according to a realistic assessment of the probable outcomes of medical treatment. These phases are clinically defined intentional categories that take heed of, but are quite distinct from, personal goals expressed by patients. Patients can move from one category to another during their illness trajectory. The phases are curative or restorative, palliative, and terminal ${ }^{1}$ they are based on phases that were first described in $1990 .^{2}$ The distinguishing features of each phase are shown in the Box.

The patient assessment is shared with the patient or substitute decisionmaker (SDM) and, if agreed, a GOC plan form is completed and placed in the alerts section of the patient's medical record. A GOC plan is a medical order that clarifies limitations of medical treatment for a present condition; it is not the same as an advance directive, which is usually made by a person, in his or her own "voice", to inform medical decision making for future episodes of impaired capacity. Goals are revised in the light of changes in medical condition, and appropriate limitations are then documented on a new form. A GOC plan replaces institutional or community-based not-for-resuscitation (NFR) orders.

We documented GOC plans using an original form (Appendix 1; online at mja.com.au), which has been used at Royal Hobart Hospital for the past 3 years. A second, revised form (Appendix 2; online at mja.com.au)

\section{Summary}

A novel clinical framework called "goals of care" (GOC) has been designed as a replacement for not-forresuscitation orders. The aim is to improve decision making and documentation relating to limitations of medical treatment.

- Clinicians assign a patient's situation to one of three phases of care - curative or restorative, palliative, or terminal - according to an assessment of likely treatment outcomes. This applies to all admitted patients, and the default position is the curative or restorative phase.

GOC helps identify patients who wish to decline treatments that might otherwise be given, such as treatment with blood products. This includes patients for whom specific limitations apply because of their beliefs.

GOC has been introduced at Royal Hobart Hospital, Tasmania, and at Northern Health, Melbourne. So far, audit data and staff feedback have been favourable. There have been no reported major incidents or complaints in which GOC has been causally implicated in an adverse outcome.

is now being introduced more widely in Tasmania, after extensive experience and feedback from clinicians, medical records staff and others. It is simpler and has been modified for use in all settings, including homes and nursing homes.

The original developmental work was done in Hobart after the Royal Hobart Hospital completed a Respecting Patient Choices pilot site project in 2008. This project put a sharp focus on decision making at the end of life across the whole hospital community.

In 2010, a project officer position was created to enable the development of GOC as part of a statewide Healthy Dying Initiative. Based on the principles of health-promoting palliative care, this initiative aimed to empower the whole community, including the health sector, to deal with death in a more direct, open and therefore "healthy" way. Clinical decision making at the end of life was identified as a priority for policy and procedural reform. There were three initial components of the Healthy Dying Initiative: GOC, advance directive redesign and promotion, and encouragement of health-promoting activities relating to death and dying.

The project officer, a non-clinician with extensive experience in community development, helped design the GOC form, develop the policy protocol for 
The three-phase model of goals of care (GOC)

\section{Curative or restorative phase} ("beating it")

The default position for all patients - all appropriate life-prolonging treatment will be deployed as indicated (Categories A and B in our forms)

\section{Palliative phase} ("living with disease, anticipating death")

The disease is deemed to be incurable and progressive (Category C in our forms)

\section{Terminal phase} ("dying very soon")

Death is believed to be imminent (ie, within a few days) - implementation of a terminal care pathway, where available, is indicated (Category $D$ in our forms)

Aim

GOC are directed towards cure, prolonged disease remission and/or restoration to the pre-episode health status for those with chronic diseases, especially in the aged care context
GOC are modified in favour of comfort, quality of life and dignity; period of survival is no longer the sole determinant of treatment choice; life prolongation is a secondary objective of medical treatment, although palliative care might confer modest survival benefits, as shown in two lung cancer studies $^{3}$

\section{Prognosis}

Life expectancy is probably indefinite (ie, normal) because the present health episode is unlikely to affect longevity; a key question could be "is there a reasonable chance of the patient leaving hospital and living the same life span as might have been expected before the episode?"; a key question in aged care and chronic disease settings (where the goals might be restorative) could be "is there a reasonable chance of the patient leaving hospital and/or returning to his or her previous level of functioning?"
Life expectancy is usually months, but sometimes years (if the latter is the case, "supportive care" might be a more appropriate term than "palliative care", and patients might choose to have active treatment of disease until disease response ceases); a key question could be "would I/we be surprised if this patient died in the next 12 months?"4
Comfort, quality of life and dignity are the only considerations
Life expectancy is hours or days; a key question could be "would I/we be surprised if this patient died this week?"

\section{Level of adverse effects}

A high level of adverse effects and even a significant chance of treatment-related mortality might be accepted for curative treatment (eg, brain aneurysm surgery, bone marrow transplant); while pain and symptom control should always be addressed, comfort may be a secondary consideration if it conflicts with curative treatment
Active treatment of the underlying disease may be undertaken for specific symptoms (eg, radiotherapy or chemotherapy for palliative end point in cancer treatment) and/or short-term life expectancy gains; treatment-related adverse effects should be proportionate to the goals and acceptable to the patient
Active treatment of the underlying disease should stop; no treatment-related toxicity is acceptable (this applies to all medical, nursing and allied health interventions [eg, turns in bed if these are distressing])

\section{Life-sustaining treatments}

Given as needed
Life-sustaining treatments for other chronic medical conditions are usually continued (eg, treatment with insulin or anticonvulsants) in cases where cessation would result in premature death or preventable unpleasant symptoms such as hyperglycaemia and seizures (ie, symptoms unrelated to the main disease that is anticipated to cause death) or where quality of life would be adversely affected ${ }^{5}$

\section{Medical provision of hydration and alimentation}

Given as needed
Given if indicated and desired (eg, percutaneous endoscopic gastrostomy feeding for head and neck cancer patients with obstructed swallowing)
Life-sustaining treatments for other chronic medical conditions are usually stopped (eg, treatment with steroids, insulin or anticonvulsants), unless doing so would cause suffering

\section{Cardiopulmonary resuscitation}

Given as needed
Usually not recommended but should be discussed with the patient, if competent; if death and dying have already been explicitly discussed with the patient or person responsible, specific discussion of cardiopulmonary resuscitation might not be warranted ${ }^{6}$
Usually ceased and replaced with feeding on request and rigorous mouth care 
its implementation and use, launch the new form, and facilitate initial training in individual hospital units. GOC education was then done jointly with the advance directive work in the wider community in collaboration with a designated officer in the Office of the Chief Health Officer, Department of Health and Human Services, Tasmania.

\section{Audit results}

On 1 March 2011, the GOC form and protocol came into effect at Royal Hobart Hospital; it replaced the NFR procedure and form, which were withdrawn with effect from that date.

A retrospective audit of admissions to the Assessment and Planning Unit during August 2011 was undertaken. It showed that GOC forms had been completed for $75 \%$ of admitted patients (135/181). A retrospective audit of admissions to the Assessment and Planning Unit during August 2009, before introduction of GOC, showed that NFR forms had been completed for $34 \%$ of admitted patients (55/162). (These data were compiled on 28 September 2009 and 26 September 2011, respectively.)

On 6 September 2012, a 1-day point prevalence audit of GOC form completion was undertaken throughout Royal Hobart Hospital, excluding paediatric and daystay patients. Patient records were reviewed for the presence of a GOC form and/or other relevant documents, such as an advance directive. GOC forms had been completed for $52 \%$ of inpatients (148/283) and for $85 \%$ of medical inpatients (124/146) who had been admitted that day. For non-medical admissions, a GOC form was completed for $21 \%$ of patients (24/112). All 18 patients who subsequently died had dying recognised (GOC category D), and half of them received input from the palliative care service.

A GOC form was implemented at Northern Health on 12 August 2013. It was adapted from the version used at Royal Hobart Hospital, using input from Northern Health clinicians. It was mandated for all adult medical inpatients and for selected surgical patients. A 1-day point prevalence audit of medical patients on 17 November 2013 showed that treatment goals were completed for $81 \%$ of patients $(82 / 101){ }^{7}$

\section{Discussion}

The purpose of GOC is to ensure that patients who are unlikely to benefit from medical treatment aimed at cure receive care appropriate to their condition and are not subjected to burdensome or futile treatments, particularly cardiopulmonary resuscitation and medical emergency team calls, especially when these are, or may be, contrary to their wishes.

One of the aims of GOC is to change the culture of medical decision making. GOC takes on the challenges of "prognostic paralysis" and the "no-surprises approach", diagnosing dying, ${ }^{8}$ and prognostic uncertainty. ${ }^{9}$ There is evidence that many decisions to limit treatment occur in crisis situations, particularly during medical emergency team calls. ${ }^{10}$ Difficult decisions therefore tend to be made after hours, in the heat of the moment, by clinicians who do not know the patient and without patient or SDM input. GOC prompts treating teams to proactively determine treatment goals at a time when the assessment is likely to be of higher quality and discussions with the patient and family are easier to arrange.

Screening all patients on admission helps identify those who wish to decline treatments that might otherwise be given to them (particularly relevant for treatments that involve blood products). Those who are fit and otherwise well can be screened with the question "are there any treatments that you do not wish to have?". Others, in light of their past history and current presentation, will require a more in-depth conversation that balances their hopes and expectations with what is medically achievable.

The default position for all patients is the curative or restorative phase, and all appropriate life-prolonging treatment should be deployed as indicated until it is clear that the clinical situation has changed. In other words, the default always favours preservation of life. It has become evident that there is an important subpopulation of patients for whom the goal is cure or restoration but specific limitations of medical treatment apply because of patient wishes or beliefs, and this is specifically articulated in GOC category B on the new Tasmanian form (Appendix 2).

GOC relies on high-quality clinical assessment and good communication skills. Most importantly, it requires clinicians to make a decision. While challenging and contested, differentiation between the palliative and terminal phases is essential. There is a large difference in the medical management and care of a person who has a potential prognosis of a year or two (eg, a patient who has incurable bone metastases due to prostate or breast cancer) and that for a person who may not survive a week.

There are many pertinent observations that can be used to diagnose dying, which can be divided into four principal domains: (i) disease activity; (ii) general functioning; (iii) specific clinical parameters; and (iv) evidence of "death talk" by patients and families. In combination, these observations can help to show whether death is anticipated within the next few days and allow a change of GOC to the terminal phase. Most of the evidence so far suggests that simple non-medical general function parameters are most predictive of impending death. ${ }^{11}$ For patients in the terminal phase, deployment of tools based on the Liverpool Care Pathway for the Dying Patient (LCP) may be considered. There has been positive experience of an LCP-type tool in Australia, ${ }^{12}$ despite some negative experiences associated with use of the LCP in the United Kingdom, for which the LCP has, perhaps unfairly, been blamed. ${ }^{13,14}$

If the diagnosis of dying is made too early and a patient's condition unexpectedly stabilises, he or she will live on provided that the care implemented is proportionate and matched to symptoms, according to 
principles presented, for example, to the Senate of Canada by the Chief Coroner of Ontario in 1997.15 There are often oscillations in patient condition as the terminal phase approaches, but, once patients are deemed to be in the terminal phase, it is unusual for them to sustainably "upgrade" back to the earlier palliative phase.

The GOC process has proved to be safe, effective and widely acceptable for addressing the limitation of medical treatment in two Australian health services that encompass large acute tertiary hospitals, with aged care and related subacute services. Feedback from clinical staff has been positive, and compliance is variable but rising. So far, there have been no reported major incidents or complaints in which GOC has been causally implicated in an adverse outcome. Comparison with the NFR era is difficult as the population denominator now consists of all admitted patients, not just those deemed unsuitable for resuscitation.

Regular review at each patient encounter is important, with changes to GOC phase and/or treatment limitations as warranted by patient wishes or condition. A clear need was identified at an early stage of the initial GOC project to ensure that limitations determined and documented during an acute admission could be continued during ambulance transfers and within homes, nursing homes and other facilities. An arbitrary 90-day endorsement validity limit was initially stipulated, but this has been removed as it was found to be unnecessary and confusing. General practitioners and community nurses were also keen to see GOC initiated in the community setting, especially for palliative care clients, and this has informed the design of the new Tasmanian form (Appendix 2).

In a recent report, the Australian Commission on Safety and Quality in Health Care acknowledged that it is necessary to attempt to reverse acute clinical deterioration but also to recognise dying and deploy appropriate palliative and terminal care. ${ }^{16}$

There were extensive discussions about patients or SDMs being required to sign the GOC form to confirm adequate consultation and agreement. The developers have resisted this, arguing that it is a medical form to direct care, and not a patient directive. The emphasis should be on a process of medical assessment and communication that ideally results in clear patient agreement, and/or consensus with the SDM and those who care for and about the person concerned, regarding any limitations of medical treatment.

A requirement for SDMs to sign a GOC form might engender guilt by conveying a false concern about the locus of responsibility for causing death. It should, however, be clear that the doctor signing the form (on behalf of the medical specialist in charge) is taking responsibility for the clinical decision and all appropriate consultation with patients or their agents, as required by ethics and law. ${ }^{17}$ Ultimately, the decision about signature requirements will lie with individual institutions and/or jurisdictions that start using GOC. Similarly, the distinction between consent and receipt of information will need to be made clear by individual institutions.

We recommend that all health care providers consider replacing their NFR procedures with the GOC approach. GOC is a solid framework for limiting medical treatment that meets the challenge for medical leadership to address the culture of death avoidance in medical decision making. ${ }^{17,18}$ It also has the potential to help address widespread professional and public concerns about bad dying. Rigorous ongoing "postmarketing" surveillance, auditing and research are, of course, necessary to ensure patient safety and transparency of process.

Acknowledgements: We thank Rob Thornton (Project Officer, Respecting Patient Choices), the other Respecting Patient Choices project team members, Jenny Fuller (Project Officer, Royal Hobart Hospital and Healthy Dying Initiative), Bruce Wilson (Project Officer, Healthy Dying Initiative), Craig White (Chief Health Officer, Department of Health and Human Services, Tasmania) and Lisa Warner (Public Guardian of Tasmania) for supporting the development of GOC and the Healthy Dying Initiative.

Competing interests: No relevant disclosures.

Provenance: Not commissioned; externally peer reviewed.

1 Ashby M, Stoffell B. Therapeutic ratio and defined phases: proposal of an ethical framework for palliative care. BMJ 1991; 302: 1322-1324.

2 Haines IE, Zalcberg J, Buchanan JD. Not-for-resuscitation orders in cancer patients - principles of decision making. Med J Aust 1990; 153: 225-229.

3 Haines IE. Managing patients with advanced cancer: the benefits of early referral for palliative care. Med J Aust 2011; 194: 107-108.

4 Boyd K, Murray SA. Recognising and managing key transitions in end of life care. BMJ 2010; 341: c4863.

5 Stevenson J, Abernethy AP, Miller C, Currow DC. Managing comorbidities in patients at the end of life. BMJ 2004; 329: 909-912.

6 Hayes B. Clinical model for ethical cardiopulmonary resuscitation decision-making. Intern Med J 2013; 43: 77-83.

7 Brimblecombe C, Crosbie D, Lim WK, Hayes B. The Goals of Patient Care project: implementing a proactive approach to patient-centred decision making. Intern Med J 2014; Jun 18 [Epub ahead of print].

8 Ellershaw J. Care of the dying: what a difference an LCP makes! Palliat Med 2007; 21: 365-368.

9 Glare P. Predicting and communicating prognosis in palliative care. BMJ 2011; 343: d5171.

10 Jones D, Moran J, Winters B, Welch J. The rapid response system and end-of-life care. Curr Opin Crit Care 2013; 19: 616-623.

$11 \mathrm{Kim}$ AS, Youn CH, Ko HJ, Kim HM. The survival time of terminal cancer patients: prediction based on clinical parameters and simple prognostic scores. J Palliat Care 2014; 30: 24-31.

12 Jackson K, Mooney C, Campbell D. The development and implementation of the pathway for improving the care of the dying in general medical wards. Intern Med J 2009; 39: 695-699.

13 Ashby M. Caring for dying patients is not about prolonging life at all costs. BMJ 2013; 346: f3027.

14 Neuberger J. More care, less pathway: a review of the Liverpool Care Pathway. 2013. https://www.gov.uk/government/uploads/system/ uploads/attachment_data/file/212450/Liverpool_Care_Pathway.pdf (accessed Sep 2014).

15 Ashby MA. On causing death. Med J Aust 2001; 175: 517-518.

16 Australian Commission on Safety and Quality in Health Care. Safety and quality of end-of-life care in acute hospitals: a background paper. Sydney: ACSQHC, 2013. http://www.safetyandquality.gov.au/ publications/safety-and-quality-of-end-of-life-care-a-backgroundpaper (accessed Jul 2014).

17 Ashby MA, Kellehear A, Stoffell BF. Resolving conflict in end-of-life care [editorial]. Med J Aust 2005; 183: 230-231.

18 Murphy BF. What has happened to clinical leadership in futile care discussions? Med J Aust 2008; 188: 418-419. 